Research Article

\title{
Stereoselective Separation and Acute Toxicity of Tau-Fluvalinate to Zebrafish
}

\author{
Qi Jia, ${ }^{1,2}$ Nana Xu, ${ }^{1,2}$ Pengqian Mu, ${ }^{1,2}$ Bo Wang, ${ }^{1,2}$ Shuming Yang, ${ }^{1,2}$ and Jing Qiu ${ }^{1,2}$ \\ ${ }^{1}$ Key Laboratory of Agro-Product Quality and Safety, Institute of Quality Standard and Testing Technology for Agro-Products, \\ Chinese Academy of Agricultural Sciences, Beijing 100081, China \\ ${ }^{2}$ Key Laboratory of Agri-Food Quality and Safety, Ministry of Agriculture, Beijing 100081, China \\ Correspondence should be addressed to Jing Qiu; qiujing@caas.cn
}

Received 13 March 2015; Revised 21 May 2015; Accepted 26 May 2015

Academic Editor: Qing X. Li

Copyright (C) 2015 Qi Jia et al. This is an open access article distributed under the Creative Commons Attribution License, which permits unrestricted use, distribution, and reproduction in any medium, provided the original work is properly cited.

\begin{abstract}
Tau-fluvalinate (TFLV) is one of the most potent chiral synthetic pyrethroids to control a wide range of pests in agricultural fields, especially in apiary. In this study, two stereoisomers of TFLV were fully separated by high-performance liquid chromatography (HPLC) with a semipreparative chiral column using cellulose-tris(3,5-dimethylphenylcarbamate) as chiral stationary phase and $n$-hexane and 2-propanol $(96 / 4, \mathrm{v} / \mathrm{v})$ as mobile phase at a flow rate of $2.5 \mathrm{~mL} \mathrm{~min}^{-1}$. The $(+)$-stereoisomer was first eluted by detecting with an optical rotation detector. After obtaining pure single stereoisomer of TFLV, acute toxicities of each isomer and TFLV standard to zebrafish were evaluated. The results showed that the (+)-stereoisomer exhibited 273.4 times higher toxicity than the (-)-stereoisomer and 6.7 times higher than TFLV standard, according to their $\mathrm{LC}_{50}$ values at $96 \mathrm{~h}$ after exposure. This indicates that the toxicity of TFLV mainly originates from (+)-stereoisomer. These results are very helpful to prepare single stereoisomer of chiral pesticides and evaluate their different toxicological effects to aquatic organisms.
\end{abstract}

\section{Introduction}

The study on chiral chemicals has become an important issue in recent years. About $25 \%$ of developed and marketed pesticides were chiral, in which at least two mirror images called enantiomers exist [1]. The first study about environmental enantioselectivity of pesticides began from the early 1990s [2]. Usually, enantiomers can exhibit different biological properties and toxicity. The active part of enantiomers would affect target species, whereas others may not. Furthermore, both of the enantiomers or one of them may give adverse effects on some nontarget species. As a result, more and more single- or enriched-enantiomer pesticides have been widely developed.

Among pesticides, synthetic pyrethroids, introduced in the early 1980s, are widely used in agriculture to control insect species [3]. Most of synthetic pyrethroids present two or three chiral centers and therefore contain two or four pairs of enantiomers or diastereoisomers. The recent studies proved that synthetic pyrethroid showed obvious enantioselectivity in environmental toxicity. For example, the (-)-enantiomer of cis-lambda-cyhalothrin (LCT) showed more acute toxicity than its antipode to zebrafish according to their lethal concentration $50\left(\mathrm{LC}_{50}\right)$ values [4]. After the zebrafish were exposed to environmental concentrations of different forms of cis-bifenthrin (BF) during the embryos developmental stage, $1 S$-cis-BF exhibited higher risk to induce oxidative stress, apoptosis, and immunotoxicity than $1 R$-cis-BF in zebrafish [5]. The results from zebrafish embryo test showed that $1 R$-cis- $\alpha$-S- and $1 R$-trans- $\alpha$-S-enantiomers of beta-cypermethrin exhibited strong developmental toxicities at concentration of $0.1 \mathrm{mg} \mathrm{L}^{-1}$, while the $1 S-c i s-\alpha-R$ - and $1 S$ trans- $\alpha$ - $R$-enantiomers induced no malformations at higher concentration (e.g., $0.3 \mathrm{mg} \mathrm{L}^{-1}$ ) [6]. After $96 \mathrm{~h}$ exposure, pyraclofos enantiomers exhibited different acute toxicity with $\mathrm{LC}_{50}$ of 2.23 and $3.99 \mathrm{mg} \mathrm{L}^{-1}$ for $R$ - and $S$-enantiomers, respectively [7].

As a chiral synthetic pyrethroid acaricide, tau-fluvalinate (TFLV, Figure 1), named as Apistan or Mavrik, has been licensed for apiculture in many countries. Since the introduction of Varroa in European colonies of honeybee, beekeepers 
<smiles>CC(C)[C@H](Nc1ccc(C(F)(F)F)cc1Cl)C(=O)O[C@@H](C#N)c1cccc(Oc2ccccc2)c1</smiles><smiles>CC(C)[C@H](Nc1ccc(C(F)(F)F)cc1Cl)C(=O)O[C@@H](C#N)c1cccc(Oc2ccccc2)c1</smiles>

FIgURE 1: Chemical structures of TFLV stereoisomers.

have had to control the number of mites to prevent colony losses [8]. The same situation has occurred in other countries in the world, including China. TFLV is a potent acaricide in controlling and treating Varroa. However, the treatments can lead to its residues in hives and environment. Haarmann et al. had studied that the queen honeybees treated with high doses of fluvalinate weighed significantly less than lowdose or control queens [9]. The residues could also result in accumulation and further influence on bee products and environmental organisms. Otherwise, United States Environmental Protection Agency (USEPA) treats TFLV as a reproductive toxin, and European Union also puts it into the list of endocrine disrupting chemicals. The solubility of TFLV is $0.002 \mathrm{mg} \mathrm{kg}^{-1}$ in water and $>1000 \mathrm{~g} \mathrm{~kg}^{-1}$ in acetone. On hydrolysis at $25^{\circ} \mathrm{C}$, its half-lives of degradation $\left(\mathrm{DT}_{50}\right)$ for a $9 \mu \mathrm{g} \mathrm{L}^{-1}$ aqueous solution were $48 \mathrm{~d}$ at $\mathrm{pH} 5,38.5 \mathrm{~d}$ at $\mathrm{pH} 7$, and $1.1 \mathrm{~d}$ at $\mathrm{pH}$ 9. Its $\mathrm{LC}_{50}$ at $96 \mathrm{~h}$ is $0.0062 \mathrm{mg} \mathrm{L}^{-1}$ for bluegill sunfish, $0.0027 \mathrm{mg} \mathrm{L}^{-1}$ for rainbow trout, and $0.0048 \mathrm{mg} \mathrm{L}^{-1}$ for carp. However, to our knowledge, little information about ecotoxicological effects of its stereoisomers is available until now.

Tau-fluvalinate is one form of racemic fluvalinate, which consists of four active diastereoisomers and is initially registered as the commercial product with the name of "fluvalinate." According to previous report $[10]$, the $(R, R)$ and $(R, S)$-isomers are mainly active in four diastereoisomers, and $(R, R)$-isomers are significantly less active than the $(R$, $S)$-isomer to both Heliothis virescens and Musca domestica. The other two diastereoisomers of fluvalinate derived from the $S$ acid are essentially inactive, so fluvalinate was renamed as tau-fluvalinate in 1994, and the manufacturer chose to support the half-resolved technical only and did not continue to support the registration of the racemic fluvalinate [11]. Two stereoisomers of tau-fluvalinate already showed different bioactivity for insects and also can exhibit different toxicity to environmental organisms. Thus, for evaluating the stereoselectivity in ecotoxicological effects, we separated and prepared these two stereoisomers using high-performance liquid chromatography (HPLC) on a commercial semipreparative chiral column and then investigated the difference of their acute toxicity to zebrafish.

\section{Materials and Methods}

2.1. Chemicals. TFLV standard (purity $>99.0 \%$ ) which consisted of two stereoisomers with equal contents was supplied by Dr. Ehrestorfer (Augsburg, Germany). Acetone, 2propanol, and $n$-hexane were obtained from Fisher Chemical
(New Jersey, USA). All of these solvents were of HPLC grade. The stock solutions of TFLV were prepared at $10000 \mathrm{mg} \mathrm{L}^{-1}$ in mobile phase.

2.2. Chromatographic Separation. Stereoisomeric separation was performed on a Waters 2695 HPLC system (Milford MA, USA). The ultraviolet (UV) wavelength was set at $254 \mathrm{~nm}$ and detected with an Agilent G1315C diode array detector (Wilmington, DE, USA). The elution orders were detected using Chiralyser-MP optical rotation detector (OR) produced by IBZ Messtechnik Company (Germany) and provided by Beijing Separation Science and Technology Development Co., Ltd. (Beijing, China), which is based on difference of refractive index between left and right linearly polarized lights. The optical signals were processed by Agilent Chemstation after signal transformation using an Agilent 35900E A/D converter. The semipreparative column (Phenomenex Lux Cellulose-1, $250 \times 10.0 \mathrm{~mm}, 5 \mu \mathrm{m}$ particle size) with chiral stationary phase (CSP) of cellulose-tris (3,5dimethylphenylcarbamate) was used. The volume of injection was $100 \mu \mathrm{L}$. Mobile phase was composed of different volume proportion of $n$-hexane and 2-propanol at the flow rate of 1.5-3.0 $\mathrm{mL} \mathrm{min}^{-1}$. The chiral column was held on room temperature (about $25^{\circ} \mathrm{C}$ ). To obtain the individual stereoisomers for the acute toxicity assay, two stereoisomers were collected at the HPLC outlet by manual operation, then evaporated to dryness, and redissolved in solvent for quantification.

2.3. Fish Husbandry and Acute Toxicity Assay. Zebrafish (Danio rerio) were obtained from the Department of Applied Chemistry of China Agricultural University. Healthy 5month-old adult fishes were selected and cultured in laboratory for more than two weeks with a $14 \mathrm{~h}$ light: $10 \mathrm{~h}$ dark cycle before the experiment to make sure that zebrafish acclimated the environment. Waters with total hardness of $250 \mathrm{mg} \mathrm{CaCO}_{3}$ per liter and with a $\mathrm{pH}$ of $7.0 \pm 0.2$ are preferable. The temperature was kept at $26 \pm 1^{\circ} \mathrm{C}$. Ten fishes ( $30 \pm 5 \mathrm{~mm}$ for length and $0.3 \pm 0.1 \mathrm{~g}$ for weight) in a glass fish $\operatorname{tank}(4 \mathrm{~L})$ and three separate tanks $(n=3)$ were tested with 5 different steady concentrations at $2.15,2.5,2.85,3.25$, and $3.75 \mu \mathrm{g} \mathrm{L}^{-1}$ for (+)-TFLV, 600, 720, 864, 1037, and $1245 \mu \mathrm{g} \mathrm{L}^{-1}$ for (-)-TFLV, and 15.52, 17.08, 18.78, 20.66, and $22.72 \mu \mathrm{g} \mathrm{L}^{-1}$ for TFLV standard, respectively.

Acetone was used to help easy dissolution of TFLV in water. Meanwhile, it was used as a control and exposed to the same concentration that was used in the most concentrated solution of the test substance. The exposure solutions were changed every day and the concentrations remained 
unchanged. The final determined concentration at $96 \mathrm{~h}$ was the level which caused $50 \%$ of the deaths of the test population $\left(\mathrm{LC}_{50}\right)$.

During the husbandry stages, the zebrafish were fed with standard diet daily until $24 \mathrm{~h}$ prior to beginning the experiment. The whole procedure for the acute toxicity test was based on [12]. These China guidelines only have a few small differences with OECD guidelines. For example, the $\mathrm{pH}$ of water was $7.8 \pm 0.2$, while it was $6-8$ in OECD guidelines. The number of test fish was at least 10, while it was at least 7 in OECD guidelines.

2.4. Data Analysis. Chromatographic parameters were calculated using the following equations: resolution factor (Rs) = $2\left(t_{2}-t_{1}\right) /\left(W_{2}+W_{1}\right)$, where $W_{1}$ and $W_{2}$ were baseline peak widths of the first and second eluted stereoisomers, respectively; capacity factor $(k)=\left(t_{R}-t_{0}\right) / t_{0}$, where $t_{0}$ was void time and was detected with 1,3,5-tri-tert-butylbenzene; separation factor $(\alpha)=k_{2} / k_{1}$, where $k_{1}$ and $k_{2}$ were capacity factors of the first and second eluted stereoisomers, respectively.

The mortality of zebrafish controlling fewer than $5 \%$ was considered valid in the whole test. And 95\% confidence limits of $\mathrm{LC}_{50}$ were performed by probit analysis. Significant differences among $\mathrm{LC}_{50}$ values presented as mean \pm standard deviation were analyzed by Student's $t$-test $(P<0.05)$. The software used for statistical analysis was SPSS 22 program.

\section{Results and Discussion}

3.1. Stereoisomeric Resolution. Traditionally, the analytical chiral column (diameter is $4.6 \mathrm{~mm}$ ) was used to investigate enantioseparations of chiral analytes with low concentration $\left(<100 \mathrm{mg} \mathrm{L}^{-1}\right)$. However, aim to obtain single stereoisomer with high purity for the following acute toxicity assay, a semipreparative chiral column with diameter of $10.0 \mathrm{~mm}$ was directly to separate two stereoisomers of TFLV in this study. The effects of many factors including different composition of mobile phase and flow rate on the stereoselective separation were investigated using a standard solution with higher concentration (10000 $\left.\mathrm{mg} \mathrm{L}^{-1}\right)$. The corresponding retention factor $(k)$, separation factors $(\alpha)$, and resolution factor (Rs) were given in Tables 1 and 2 .

From Table 1 it can be seen that when 2-propanol content increased from $2 \%$ to $10 \%, k, \alpha$, and Rs decreased but Rs was more apparent than $\alpha$. Rs descended from 2.204 to 1.033, and the reason may be due to different influences of 2-propanol on interaction between two stereoisomers and CSP, although $\alpha$ decreased with the increase of 2-propanol, which is helpful for preparation because this can accelerate stereoisomeric elution and shorten the retention time at the same time. As shown in Table 2, when the flow rate increased from 1.5 to $3.0 \mathrm{~mL} \mathrm{~min}^{-1}$, Rs decreased from 2.135 to 1.471 , which may be caused by accelerating elution of stereoisomers from CSP and leading to shorter interaction time between stereoisomers and CSP. However, $\alpha$ varied between 1.324 and 1.390 that nearly had no obvious change with increase of flow rate,
TABLE 1: Effect of 2-propanol content on the stereoselective separation of TFLV ${ }^{\mathrm{a}}$.

\begin{tabular}{lcccc}
\hline $\begin{array}{l}\text { 2-Propanol } \\
\text { content (\%) }\end{array}$ & $k_{1}$ & $k_{2}$ & $\alpha$ & Rs \\
\hline 2 & 4.717 & 6.923 & 1.468 & 2.204 \\
4 & 3.110 & 4.272 & 1.374 & 1.674 \\
6 & 2.513 & 3.260 & 1.297 & 1.337 \\
8 & 2.145 & 2.665 & 1.243 & 1.142 \\
10 & 1.864 & 2.265 & 1.215 & 1.033 \\
\hline
\end{tabular}

${ }^{\mathrm{a}} t_{0}$ is $4.919 \mathrm{~min}$. The flow rate was $2.5 \mathrm{~mL} \mathrm{~min}^{-1}$.

TABLE 2: Effect of flow rate on the stereoselective separation of TFLV $^{\mathrm{a}}$.

\begin{tabular}{lccccc}
\hline $\left.\begin{array}{l}\text { Flow rate } \\
(\mathrm{mL} \mathrm{min}\end{array} \mathrm{min}^{-1}\right)$ & $t_{0}(\mathrm{~min})$ & $k_{1}$ & $k_{2}$ & $\alpha$ & Rs \\
\hline 3.0 & 4.098 & 3.168 & 4.195 & 1.324 & 1.471 \\
2.5 & 4.919 & 3.110 & 4.272 & 1.374 & 1.674 \\
2.0 & 6.005 & 3.294 & 4.405 & 1.337 & 1.755 \\
1.5 & 8.167 & 3.760 & 5.226 & 1.390 & 2.135 \\
\hline
\end{tabular}

${ }^{a}$ The mobile phase is $n$-hexane/2-propanal $(96 / 4, v / v)$.

indicating that the relative retention of two stereoisomers on CSP was invariable under this condition.

According to the above results, to reduce the usage mount of the solvent and shorten preparation time, the optimum resolution condition of TFLV stereoisomers on the semipreparative column was selected with a mobile phase of $n$-hexane/2-propanal $(96 / 4, \mathrm{v} / \mathrm{v})$ at a flow rate of $2.5 \mathrm{~mL} \mathrm{~min}^{-1}$ (typical chromatogram was shown in Figure 2). The optical purity of single isomer was found to be $>99.0 \%$ for both stereoisomers collected from chiral HPLC. The elution orders of two stereoisomers were identified using the online OR. The first eluted stereoisomer showed right (+)-rotation signal but left (-)-signal for the second eluted stereoisomer. Furthermore, it is interesting that $(+)$-stereoisomer exhibited obviously higher OR signal compared to (-)-stereoisomer although they had nearly the same UV signal (Figure 2). The reason may be that these two stereoisomers from TFLV are a pair of diastereoisomers but not enantiomers, and generally only two enantiomers in racemic analyte can exhibit equivalent left (-)- or right (+)-rotation signals. Thus, TFLV is only a mixture of $(+)$ - and (-)-stereoisomer but not racemic because of different optical signals of two stereoisomers. Additionally, the changes of mobile phase and flow rate would not lead to changes of stereoisomeric elution orders of TFLV, indicating stable chiral discrimination interactions between stereoisomers and CSP.

3.2. Stereoselective Acute Toxicity to Zebrafish. Acute toxicity to zebrafish was investigated for both individual stereoisomers and the standard of TFLV through $96 \mathrm{~h}$ static tests. Zebrafish showed very different movements in different groups processed with three forms of TFLV, respectively. The fishes exhibited the strongest reaction including the violent shaking and fast swimming when (+)-TFLV was added in the 

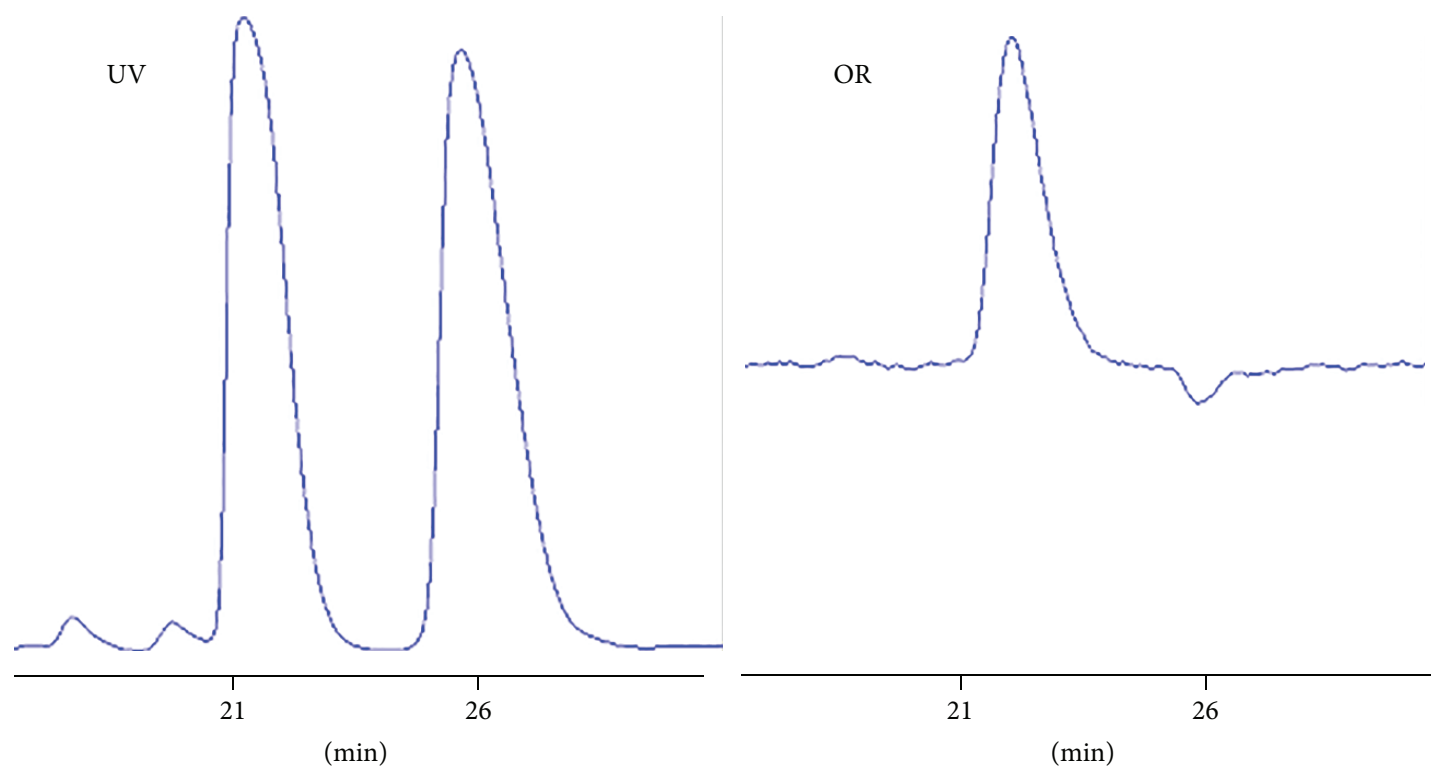

FIGURE 2: The typical UV and OR chromatograms of TFLV on semipreparative chiral column with $n$-hexane/2-propanol (96/4, v/v) at $2.5 \mathrm{~mL} \mathrm{~min}^{-1}$.

water. However, no similar phenomenon was observed in the group using (-)-TFLV, and the fishes using TFLV standard only exhibited an active swimming but not strong reaction compared to that using (+)-TFLV.

The two stereoisomers presented a significant difference in their $\mathrm{LC}_{50}$ with the lowest value of $2.5 \pm 0.2 \mu \mathrm{g} \mathrm{L}{ }^{-1}$ for (+)-TFLV compared to those for (-)-TFLV and the TFLV standard after a $96 \mathrm{~h}$ exposure (Table 3 ). This indicates that the acute toxicity of (+)-TFLV was the highest to zebrafish and about 273.4 and 6.7 times of (-)-TFLV and the standard, respectively. The presence of (-)-TFLV in the standard significantly reduced toxicity of (+)-TFLV because the $\mathrm{LC}_{50}$ of TFLV was between those of two stereoisomers and 40.9 times higher than that of (-)-TFLV. This means that an optical pure pesticide which consisted of single (-)-TFLV may be a better selection for replacement of TFLV as it exhibited the lowest acute toxicity compared to (+)-TFLV and TFLV standard, which can obviously decrease environmental toxicity of TFLV to aquatic organisms. The toxic differences of TFLV are similar to the results of other insects in Anderson et al's report [10]. They found that $(R, S)$-stereoisomer of fluvalinate is significantly more active than $(R, R)-,(S, S)$-, and $(S, R)$-stereoisomers to both Heliothis virescens and Musca domestica. Thus, comparing the testing results in toxicity, we infer that (+)-TFLV and (-)-TFLV in our study could possess configurations of $(R, S)$ and $(R, R)$, respectively. However, this inference needs to be further confirmed by other experiments in the future.

In some other studies about the chiral pyrethroid pesticides, the stereoisomers also showed the different toxicity with their racemates. Fenvalerate (FV) is one of the most potent pyrethroid insecticides to control a wide range of insect pests in agricultural fields, and the different toxicities of 4 stereoisomers to zebrafish were observed with orders of FV4 $<\mathrm{FV} 1<\mathrm{FV} 3<\mathrm{FV} 2$ according to $\mathrm{LC}_{50}$ values. FV4 showed 17 ,
TABLE 3: $L_{50}$ values of different forms of TFLV at $96 \mathrm{~h}$ after exposure to zebrafish ${ }^{\mathrm{a}}$.

\begin{tabular}{lcc}
\hline Compound & $\mathrm{LC}_{50}\left(\mu \mathrm{g} \mathrm{L}^{-1}\right)$ & $\begin{array}{c}95 \% \text { confidence } \\
\text { interval }\left(\mu \mathrm{g} \mathrm{L}^{-1}\right)\end{array}$ \\
\hline TFLV & $16.7 \pm 0.4^{\mathrm{b}}$ & $14.5-17.9$ \\
$(+)-\mathrm{TFLV}$ & $2.5 \pm 0.2^{\mathrm{c}}$ & $2.2-2.7$ \\
$(-)-\mathrm{TFLV}$ & $683.6 \pm 26.2^{\mathrm{d}}$ & $563.5-762.6$ \\
\hline
\end{tabular}

${ }^{\mathrm{a}}$ Different letters indicate significant difference by Student's $t$-test $(P<0.05)$.

22, 40, and 55 times higher toxicity than FV2 at $24 \mathrm{~h}, 48 \mathrm{~h}$, $72 \mathrm{~h}$, and $96 \mathrm{~h}$, respectively [2]. The $\mathrm{LC}_{50}$ of (-)-enantiomer of lambda-cyhalothrin was 162 times higher than that of (+)-enantiomer after $96 \mathrm{~h}$ exposure [13]. In the toxic studies of cis-bifenthrin and cis-permethrin, both C. dubia and D. magna were used in the tests. The $\mathrm{LC}_{50}$ of $1 R$-cis-enantiomer was 15-38 times more active than the corresponding $1 S$-cisenantiomer for both pesticides, while for trans-permethrin, much higher toxicity was found for the $1 R$-trans-enantiomer than the $1 S$-trans-enantiomer, with difference of at least 2030 times about their $\mathrm{LC}_{50}$ [14]. For cypermethrin, only two of eight stereoisomers, $1 R$-cis- $\alpha S$ - and $1 R$-trans- $\alpha S$-isomers, were found to possess main activity of the racemate against C. dubia. For cyfluthrin, the $1 R$-cis- $\alpha S$ - and $1 R$-trans- $\alpha S$ isomers were 50-100 times more toxic to C. dubia than the other stereoisomers [15]. Compared to these chiral pesticides, two stereoisomers of TFLV showed the larger differences (273.4 times) in toxicity. According to [12] in China, both (+)TFLV and TFLV standard belong to the hypertoxic pesticide because their $\mathrm{LC}_{50}$ values at $96 \mathrm{~h}$ were $<0.1 \mathrm{mg} \mathrm{L}^{-1}$, while (-)-TFLV is a highly toxic pesticide. This indicated that it is very necessary to separate them and evaluate ecotoxicological effects to nontarget environments and bioactivity of individual stereoisomer to target organisms. 


\section{Conclusion}

Two stereoisomers of TFLV were successfully separated on a cellulose-based semipreparative chiral column in normal phase mode. The lower content of 2-propanol and flow rate can result in better separations, and then an optimal chromatographic condition was selected for preparation of optical pure stereoisomers. The results of exposure experiment to zebrafish showed that there were some significant differences among acute toxicity of individual stereoisomers and their mixture. The (+)-stereoisomer of TFLV presented the highest toxicity to zebrafish compared to (-)-stereoisomer and the TFLV standard. These results are helpful to obtain single stereoisomers of chiral pyrethroid pesticides and study their stereoselective behaviors in environment and ecosystem.

\section{Conflict of Interests}

The authors declare that there is no conflict of interests regarding the publication of this paper.

\section{Acknowledgment}

This work was funded by the National Natural Science Foundation of China (nos. 21177156 and 20907073).

\section{References}

[1] A. W. Garrison, "Probing the enantioselectivity of chiral pesticides," Environmental Science and Technology, vol. 40, no. 1, pp. 16-23, 2006.

[2] Y. Ma, L. Chen, X. Lu, H. Chu, C. Xu, and W. Liu, "Enantioselectivity in aquatic toxicity of synthetic pyrethroid insecticide fenvalerate," Ecotoxicology and Environmental Safety, vol. 72, no. 7, pp. 1913-1918, 2009.

[3] Z. M. Chen and Y. H. Wang, "Chromatographic methods for the determination of pyrethrin and pyrethroid pesticide residues in crops, foods and environmental samples," Journal of Chromatography A, vol. 754, no. 1-2, pp. 367-395, 1996.

[4] J. Ye, M. Zhao, J. Liu, and W. Liu, "Enantioselectivity in environmental risk assessment of modern chiral pesticides," Environmental Pollution, vol. 158, no. 7, pp. 2371-2383, 2010.

[5] Y. Jin, X. Pan, L. Cao, B. Ma, and Z. Fu, "Embryonic exposure to cis-bifenthrin enantioselectively induces the transcription of genes related to oxidative stress, apoptosis and immunotoxicity in zebrafish (Danio rerio)," Fish and Shellfish Immunology, vol. 34, no. 2, pp. 717-723, 2013.

[6] C. Xu, W. Tu, C. Lou, Y. Hong, and M. Zhao, "Enantioselective separation and zebrafish embryo toxicity of insecticide betacypermethrin," Journal of Environmental Sciences, vol. 22, no. 5, pp. 738-743, 2010.

[7] S. Zhuang, Z. Zhang, W. Zhang, L. Bao, C. Xu, and H. Zhang, "Enantioselective developmental toxicity and immunotoxicity of pyraclofos toward zebrafish (Danio rerio)," Aquatic Toxicology, vol. 159, pp. 119-126, 2015.

[8] M. P. Chauzat and J. P. Faucon, "Pesticide residues in beeswax samples collected from honey bee colonies (Apis mellifera L.) in France," Pest Management Science, vol. 63, no. 11, pp. 1100-1106, 2007.
[9] T. Haarmann, M. Spivak, D. Weaver, B. Weaver, and T. Glenn, "Effects of fluvalinate and coumaphos on queen honey bees (Hymenoptera: Apidae) in two commercial queen rearing operations," Journal of Economic Entomology, vol. 95, no. 1, pp. 28-35, 2002.

[10] R. J. Anderson, K. G. Adams, and C. A. Henrick, "Synthesis and insecticidal activity of the stereoisomers of $\alpha$-cyano-3phenoxybenzyl 2-[2-chloro-4-(trifluoromethyl)anilino]-3methylbutanoate (fluvalinate) and related analogues," Journal of Agricultural and Food Chemistry, vol. 33, no. 3, pp. 508-514, 1985.

[11] D. Edwards, Reregistration Eligibility Decision for TauFluvalinate, 2005.

[12] Ministry of Agriculture of the People's Republic of China, GB/T 31207.12-2014 Guidelines on Environmental Safety Assessment for Chemical Pesticides-Section 12: Fish Acute Toxicity Test, Ministry of Agriculture of the People's Republic of China, Beijing, China, 2014.

[13] C. Xu, J. J. Wang, W. P. Liu, G. D. Sheng, Y. J. Tu, and Y. Ma, "Separation and aquatic toxicity of enantiomers of the pyrethroid insecticide lambda-cyhalothrin," Environmental Toxicology and Chemistry, vol. 27, no. 1, pp. 174-181, 2008.

[14] W. Liu, J. J. Gan, and S. Qin, "Separation and aquatic toxicity of enantiomers of synthetic pyrethroid insecticides," Chirality, vol. 17, supplement, pp. S127-S133, 2005.

[15] W. P. Liu, J. Y. Gan, D. Schlenk, and W. A. Jury, "Enantioselectivity in environmental safety of current chiral insecticides," Proceedings of the National Academy of Sciences of the United States of America, vol. 102, no. 3, pp. 701-706, 2005. 

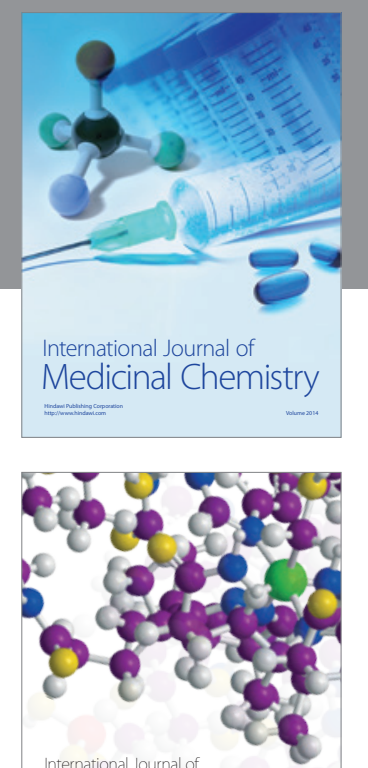

\section{Carbohydrate} Chemistry

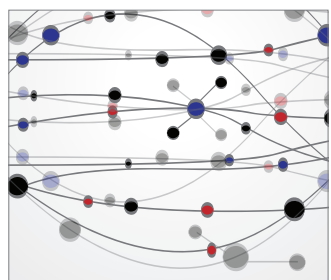

The Scientific World Journal
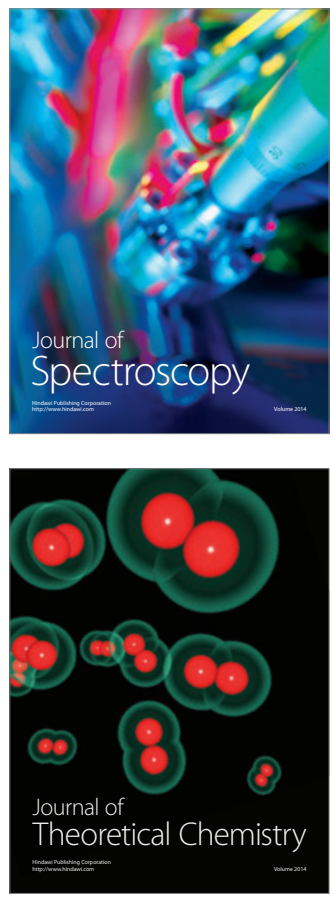
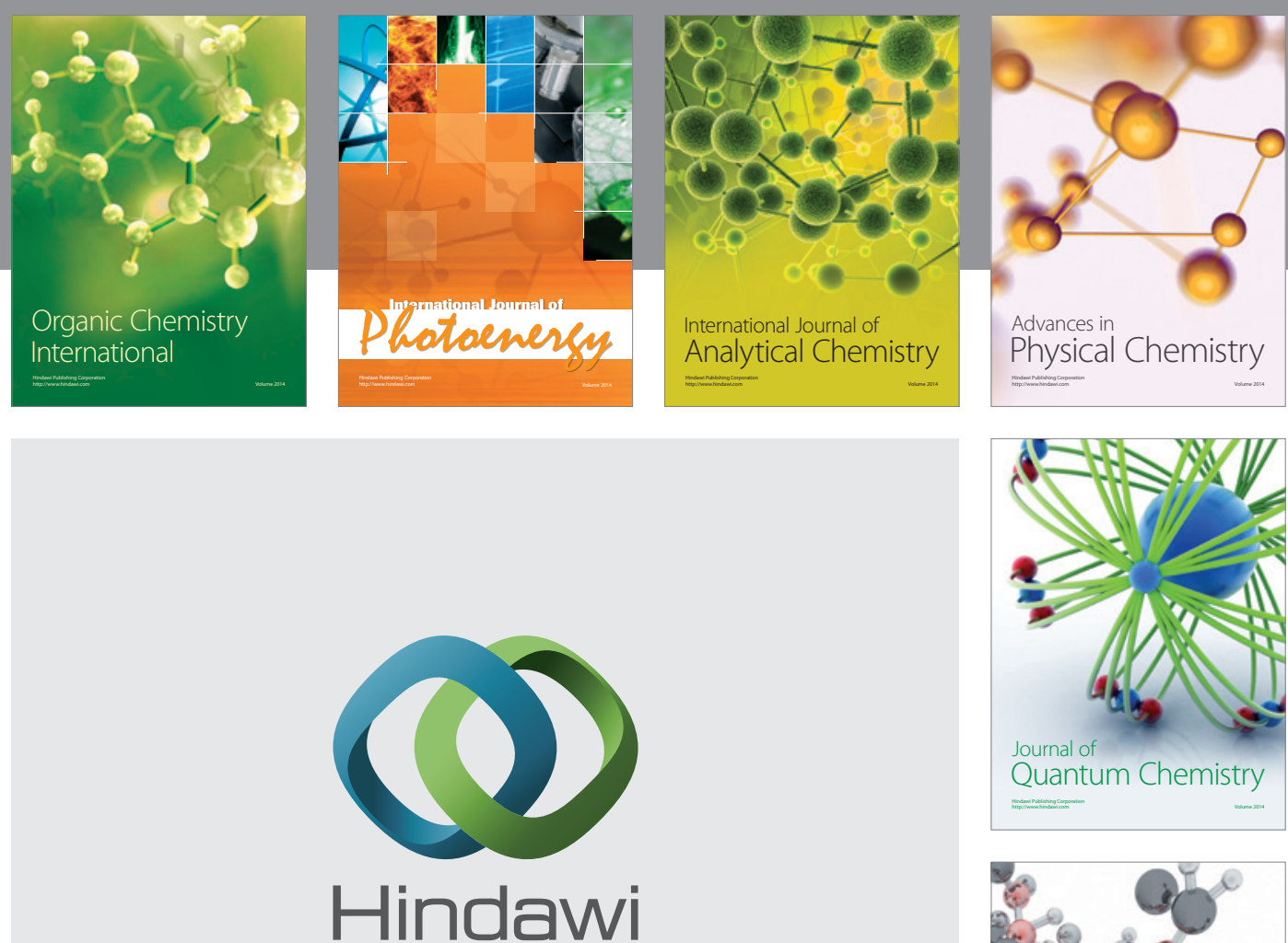

Submit your manuscripts at

http://www.hindawi.com

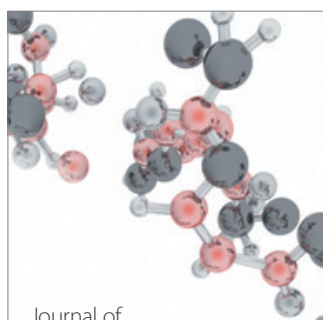

Analytical Methods

in Chemistry

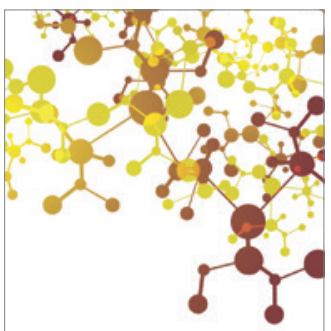

Journal of

Applied Chemistry

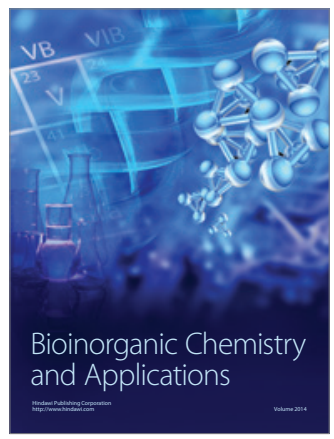

Inorganic Chemistry
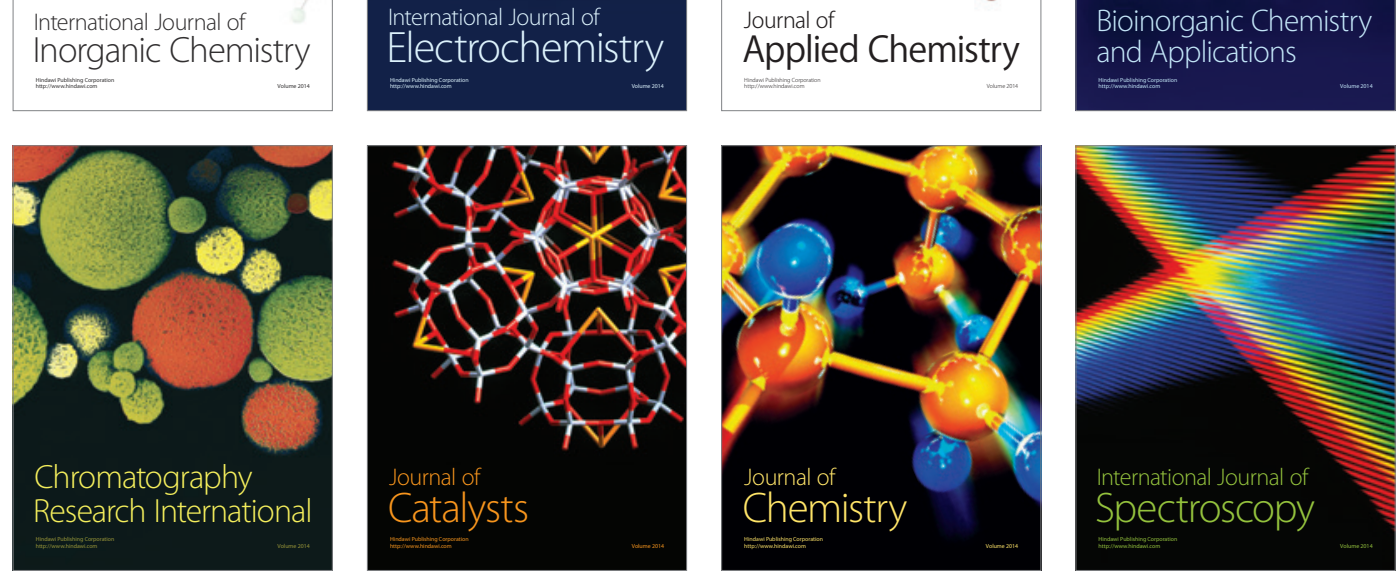